\title{
Cutaneous Metaplastic Synovial Cyst
}

"Sunitha Ramachandra, ${ }^{1}$ Lakshmi Rao, ${ }^{2}$ Masoud Al-Kindi ${ }^{2}$

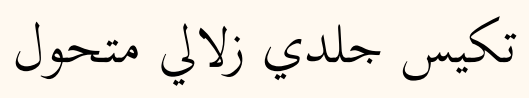

سونيثا راماشاندرا، لاكثمي راو، مسعود الكندي

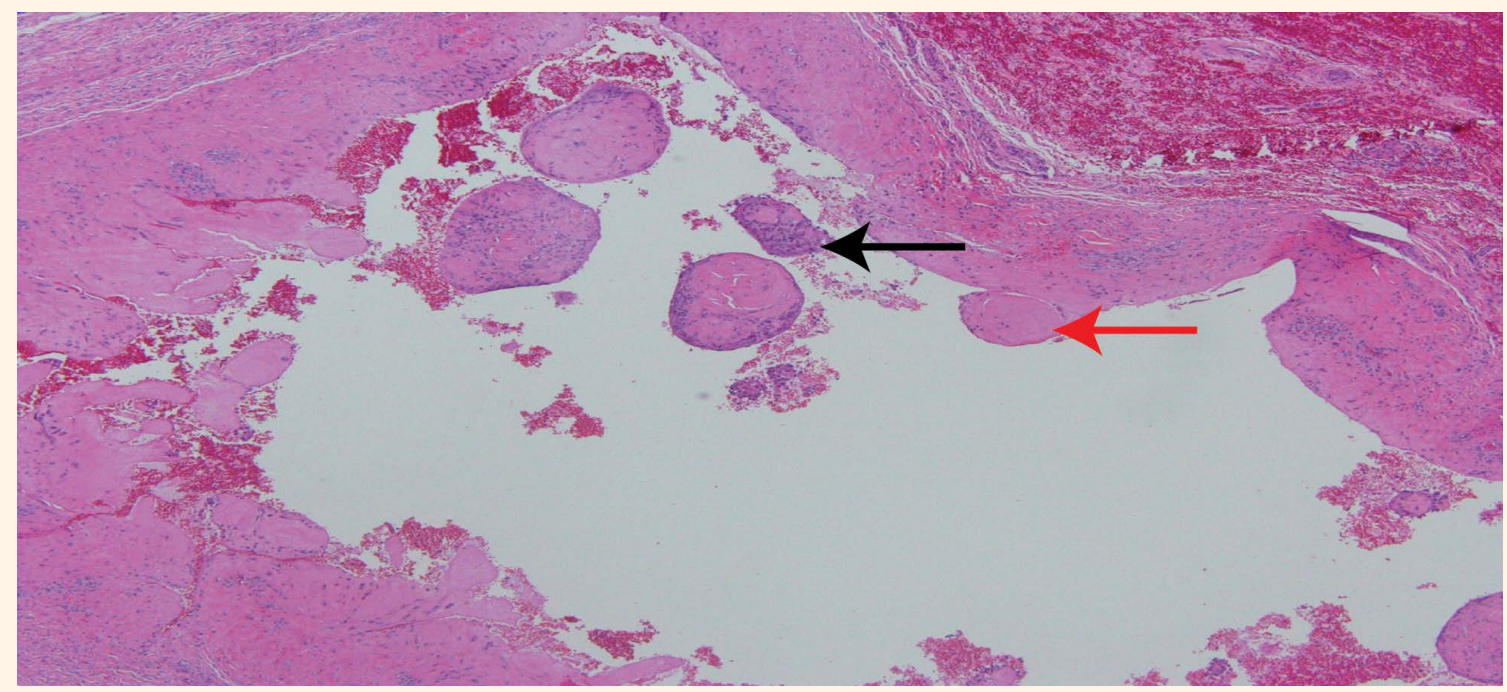

Figure 1: Haematoxylin and eosin stain at $\mathrm{x} 4$ magnification showing the cyst wall with broad papillae and villi lined by synoviocytes (red arrow) with hyalinised cores (black arrow) in a 31-year-old patient with a cutaneous metaplastic synovial cyst.

A 31-YEAR-OLD MALE PATIENT PRESENTED to the surgical outpatient department at the Armed Forces Hospital, Muscat, Oman, in September 2014 with a swelling on the left side of his forehead that had been on-going for 10 months. A clinical examination showed a subcutaneous, mobile and non-tender swelling. He had a past history of a similar swelling at the same site, which had been operated on four years previously at another hospital. No reports were available regarding the nature of this previous swelling.

At presentation, the swelling was diagnosed clinically as a recurrent dermoid cyst and was excised and sent for histopathological examination. The pale grey firm nodular mass $(1.2 \times 1.0 \times 0.8 \mathrm{~cm})$ showed a solid cystic mass when sliced. An irregular cyst lined by hyperplastic synoviocytes forming broad villi and papillae with hyalinised cores infiltrated by lymphocytes and plasma cells was observed on microscopic examination [Figure 1]. Foci of chond- roid metaplasia, haemorrhage and pigment-laden macrophages surrounded by skeletal muscle with atrophic changes and giant cell formation were noted. A histopathological diagnosis of cutaneous metaplastic synovial cyst (CMSC) was made. Immunohistochemistry (IHC) showed vimentinpositive [Figure $2 \mathrm{~A}]$ and cluster of differentiation $(\mathrm{CD})$ 68-positive [Figure 2B] synoviocytes. However, IHC was negative for pan-cytokeratin and CD34.

\section{Comment}

Also known as synovial metaplasia of the skin, CMSC is a rare cystic tumour first described in 1987 by Gonzalez et al. ${ }^{1}$ The tumour is located intradermally or subcutaneously and is lined by a hyperplastic synovium-like membrane associated with transepidermal fistulae. ${ }^{2}$ The aetiology remains unknown, although a history of trauma or surgery is noted in most cases, suggesting that surgical or 

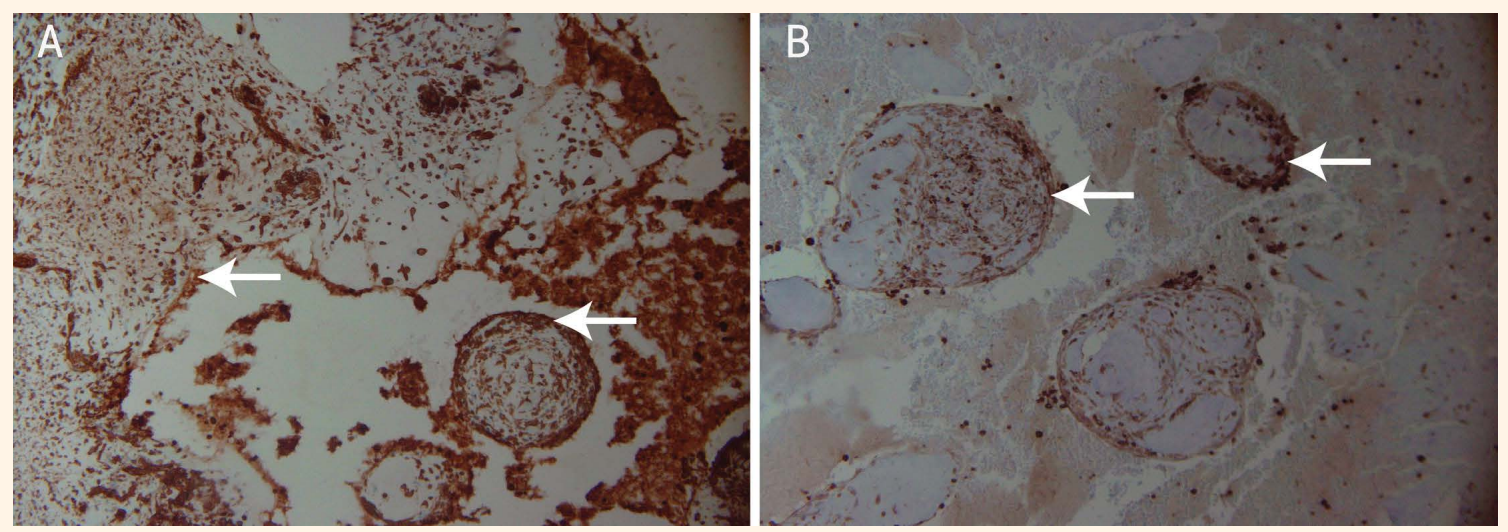

Figure 2A \& B: Immunohistochemistry stains at x20 magnification showing (A) vimentin-positive (arrows) and (B) cluster of differentiation 68-positive (arrows) synoviocytes in a 31-year-old patient with a cutaneous metaplastic synovial cyst.

non-surgical trauma is the precipitating cause. ${ }^{3}$ The spontaneous development of these cysts in Ehlers-Danlos syndrome indicates the occurrence of cutaneous fragility and anomalous scarring after microtrauma. ${ }^{4}$ Cases of CMSC have been associated with basal cell carcinomas, rheumatoid arthritis and arthrosis. ${ }^{3}$ Within the embryo, the normal synovium develops from a mechanical disruption of the connective tissue; CMSCs form due to the disruption of connective tissue following trauma or surgery. ${ }^{5}$ The cyst develops between a few weeks to several years following the trauma. It has no predilection for site, gender or age. ${ }^{5}$ Clinically, CMSCs are tender, erythematous, cutaneous or dermal nodules with or without drainage. ${ }^{3}$

CMSC remains a lesser known entity in clinical dermatology. ${ }^{5}$ It is commonly misdiagnosed and the lack of clinical awareness is an impediment to establishing its true incidence. The swelling is commonly misdiagnosed as a suture granuloma or an epidermal inclusion cyst following surgery or trauma. ${ }^{5}$ Other differential diagnoses include foreign body granulomas, leiomyomas, eccrine poromas and other cutaneous cysts. ${ }^{3}$ Epidermal cysts are distinguished from CMSCs as they are true cysts with an epithelial lining. In comparison, CMSCs lack a true epithelial lining and are pseudocysts with papillary and villous projections towards the centre of the cavity. The lining is similar to a synovial membrane and is composed of hypo- and hypercellular areas with a mixture of fibroblasts, epithelioid cells, mononuclear inflammatory cells and occasional multinucleated giant cells. ${ }^{1,3}$ Hypocellular areas show fibrin deposits and hyalinisation. When located close to a joint cavity, these lesions need to be differentiated from the fluid-filled sacs of bursitis, which are deeper in the subcutaneous tissue and located over the bony prominences. ${ }^{1}$ IHC testing in CMSC cases shows positive staining for vimentin and negative staining for the S100 protein, carcinoembryonic antigen and CD34. CD68 shows a variable reaction. However, IHC is not mandatory for the diagnosis, as CMSC can be diagnosed histologically. ${ }^{3}$

\section{References}

1. Kim BC, Choi WJ, Park EJ, Kwon IH, Cho HJ, Kim KH, et al. Cutaneous metaplastic synovial cyst of the first metatarsal head area. Ann Dermatol 2011; 23:S165-8. doi: 10.5021/ad.2011.23. S2.S165.

2. Rosai J. Rosai and Ackerman's Surgical Pathology. 9th ed. St Louis, Missouri, USA: Mosby, 2004. Pp. 202.

3. Yang HC, Tsai YJ, Hu SL, Wu YY. Cutaneous metaplastic synovial cyst: A case report and review of literature. Dermatol Sinica 2003; 21:275-9.

4. Weedon D. Weedon's Skin Pathology. 3rd ed. London, UK: Churchill Livingstone, 2010. Pp. 454.

5. Shim WH, Jwa SW, Song M, Kim HS, Ko HC, Kim BS, et al. Cutaneous metaplastic synovial cyst of the cheek generated by repetitive minor trauma. Ann Dermatol 2011; 23:S235-8. doi: $10.5021 /$ ad.2011.23.S2.S235. 